\title{
Acute Respiratory Syndrome and Pneumonia caused by COVID-19: A Systematic Review and Meta-Analysis
}

\author{
Attapon Cheepsattayakorn ${ }^{1 *}$ and Ruangrong Cheepsattayakorn ${ }^{2}$ \\ ${ }^{1} 10$ th Zonal Tuberculosis and Chest Disease Center, Thailand \\ ${ }^{2}$ Department of Pathology, Faculty of Medicine, Chiang Mai University, Thailand
}

*Corresponding author: Attapon Cheepsattayakorn, $10^{\text {th }}$ Zonal Tuberculosis and Chest Disease Cher, 143 Sridornchai Road Changklan Muang Chiang Mai 50100 Thailand, Tel: 6653 140767; 6653 276364; Fax : 6653 140773; 6653 273590; Email : Attapon1958@gmail.com

\section{Abstract}

Objectives: The objectives of this study are to identify sources of the transmission, the starting date of outbreak, epidemic trend of the COVID-19 in China, risk of global spread, case definition for surveillance, detection, prevention, control, diagnosis, and treatment options.

Study Method: A comprehensive search was carried out in mainstream bibliographic databases or Medical Subject Headings, including ScienDirect, PubMed, Scopus, and ISI Web of Science, including the websites of the news on the google scholar. The search was applied to the articles that were published between 2003 and February 2020.

Study Results: With strict literature search and screening processes, it yielded 32 articles from 366 articles of initial literature database and websites of the news. Coronavirus were found in the mid-1960s that can infect both humans and animals (birds and mammals), whereas seven coronaviruses are known to infect humans, such as Betacoronavirus HCoV-OC43 and HCoVHKU1 and Alphacoronavirus HCoV-229E. These coronaviruses primarily target on epithelial cells in the respiratory and gastrointestinal tracts through various routes of transmission, such as respiratory droplets, airborne, fecal-oral or fomites. On December 31, 2019, the Wuhan Municipal Health Commission in Wuhan City, Hubei province, China reported a cluster of 27 pneumonia cases of unknown etiology, including 7 severe cases, with a common reported connection with Wuhan's Huanan Seafood Wholesale Market (a wholesale fish and live animal market selling different animal species). These cases presented with several infectious respiratory disease, such as fever, dyspnea, and bilateral pulmonary infiltrates on chest roentgenograms. Nevertheless, on January 19, 2020, first COVID-19-infected case of 35-year-old man presented to an urgent care clinic in Snohomish County, Washington was detected and reported in the United States. Intravenous remdesivir (a novel nucleoside analogue prodrug in development) was administrated on the evening of day 7 of hospitalization due to the patient's ongoing fevers, the persistent positive COVID-19 RNA at multiple sites, and notification of rales in both lungs at a period consistent with the development of roentgenographic pneumonia. On hospital day 8 (illness day 12), there is improvement of the patient's clinical condition. Another recent study on COVID-19 inhibition in vitro by remdesivir and chloroquine demonstrated that these drugs were highly effective in the control of COVID-19 infection in vitro. These drugs should be evaluated in humans infected with COVID-19.

Conclusion: Without implementation of proper infection prevention and control measures at the point of care for individuals under investigation, there will be a likelihood of disease outbreaks, particularly via traveler transmission, transmission on aircrafts, and healthcare-related transmission in the destination countries. Further investigations for COVID-19 are urgently needed due to its potentially global health threat.

Keywords: Case definition; COVID-19; Epidemic; Outbreak; Surveillance; Wuhan; China; Respiratory; Pneumonia; Prevention; Control; Diagnosis; Treatment 


\section{Open Access Journal of Pulmonary \& Respiratory Sciences}

Abbreviations: ACE: Angiotensin-Converting Enzyme; CCDC: Chinese Center for Disease Control and Prevention; CI: Confidential Interval; COVID-19: Coronavirus Disease-2019; GCSF: Granulocyte-Colony-Stimulating Factor, IL: Interleukin; IP: Interferon Gamma-Induced Protein; IQR: Interquatile Range; MCP: Monocyte Chemoattractant Protein; MERS: Middle-East Respiratory Syndrome; MIP: Macrophage Inflammatory Protein; $\mathrm{R}_{0}$ : Basic Reproductive Number; $\mathrm{R}_{1}$ : Effective Reproductive Number; RNA: Ribonucleic Acid, RTPCR: Reverse Transcriptase-Polymerase Chain Reaction, SARS: Severe Acute Respiratory Syndrome, TNF: Tumor Necrosis Factor, WHO: World Health Organization.

\section{Objectives of the Study}

The objectives of this study are to identify sources of the transmission, the starting date of outbreak, epidemic trend of the COVID-19 in China, risk of global spread, case definition for surveillance, detection, prevention, control, diagnosis, and treatment options.

\section{Method of the Study}

A comprehensive search was carried out in mainstream bibliographic databases or Medical Subject Headings, including ScienceDirect, PubMed, Scopus, and ISI Web of Science, including the websites of the news on the google scholar. The search was applied to the articles that were published between 2003 and February 2020. Our first involved performing searches of article abstract/ keywords/title using strings of [("novel 2019-coronavirus" or "Wuhan virus" and "COVID-19" or "2019-nCoV"]. After a first approach of search, published articles focusing on outbreaks, risk of global spread, diagnostic methodology, and therapeutic interventions of COVID-19 were retained and the information on COVID-19 was extracted for having a crude knowledge involving their themes. Another round of publication search was conducted for adding the missing published articles that were not identified by the first round.

All keywords combinations from COVID-19 and 2019$n \mathrm{CoV}$ outbreak to bind the population of cases under consideration. The initial literature databases were further manually screened with the following rules: 1) non-COVID-19 -related articles were excluded; 2) articles that did not report an outbreak related to COVID-19 were not considered, such as commentary articles, or editorial; 3) non-peer reviewed journal articles were not considered to be of a scholarly trustworthy validity; and 4) duplicated and non-English articles were removed. The articles were carefully selected to guarantee the literature quality, which is a trade-off for quantity.

\section{Results}

With strict literature search and screening processes, it yielded 32 articles from 366 articles of initial literature database and websites of the news. Needed article information was extracted from each article by: 1) direct information including journal, title, authors, abstract, full text documents of candidate studies, news on the websites, publishing year; 2) place name of the study area; 3) study period; 4) research method used.

\section{Introduction and Epidemiological Background}

Coronavirus were found in the mid-1960s that can infect both humans and animals (birds and mammals), whereas seven coronaviruses are known to infect humans, such as Betacoronavirus $\mathrm{HCoV}-\mathrm{OC} 43$ and HCoV-HKU1 and Alphacoronavirus HCoV-229E. These coronaviruses primarily target on epithelial cells in the respiratory and gastrointestinal tracts through various routes of transmission, such as respiratory droplets, airborne, fecal-oral or fomites. These coronaviruses cause common colds as well as severe lower respiratory tract infections in the youngest and oldest age groups [1].

Wuhan, a city of more than 11 million residents is connected to other cities in China via high-speed railway and commercial airline flights. During January 2017 (as of January 31,2020 ), there were 670,417 passenger bookings departing Wuhan, the top destinations being Shanghai of 53,214 bookings, Beijing of 51,066 bookings, and Kunming of 40,120 bookings [2]. Wuhan is connected internationally via both direct and indirect flights [3]. COVID-19 was first identified from a patient with pneumonia, related to the cluster of acute respiratory illness cases from Wuhan, China with close relation to SARS-CoV and genetically clusters within the genus Betacoronavirus, subgenus Sarbecovirus [4]. With bases on the epidemiological characteristics of respiratory infections caused by SARS-CoV and MERS-CoV, its incubation period of 2 to 7 days and up to 14 days is possible. Approximately $20 \%$ of the laboratory-confirmed cases are seriously or critically ill and at least 4 confirmed cases have died [5]

On December 31, 2019, the Wuhan Municipal Health Commission in Wuhan City, Hubei province, China reported a cluster of 27 pneumonia cases of unknown etiology, including 7 severe cases, with a common reported connection with Wuhan's Huanan Seafood Wholesale Market (a wholesale fish and live animal market selling different animal species) [6]. These cases presented with several infectious respiratory disease, such as fever, dyspnea, and 


\section{Open Access Journal of Pulmonary \& Respiratory Sciences}

bilateral pulmonary infiltrates on chest roentgenograms. Chinese authorities placed all cases under isolation, initiated contact tracing activities and hygiene and environmental sanitation activities at this market, which was closed to the public on January 1,2020 . At that time (as of January 1, 2020), no significant human-to-human transmission and no cases among healthcare workers were reported by the Chinese authorities. Between December 31, 2019 and January 20, 2020, 295 COVID-19-laboratory-confirmed cases, including 4 deaths, have been reported [7]. During this period (December 31, 2019-January 20, 2020), of the 295 laboratory-confirmed cases, 291 cases were reported by China (270 cases in Wuhan City, 5 cases in Beijing, 14 cases in Guangdong, and 2 cases in Shanghai) [7]. Fifteen healthcare workers in Wuhan were the reported cases during that period [8]. During that period (December 31, 2019-January 20, 2020), Wuhan City reported that 169 cases were hospitalized, of which 35 cases were seriously and 9 cases were critically ill [9]. During the period between January 9 , 2020 and January 19, 2020, in Guangdong, China, 2 of the 14 reported cases had not travelled to Wuhan, China, but had a history of contact with laboratory-confirmed cases were the first confirmed human-to-human transmission cases [10], whereas the other four laboratory-confirmed cases were outside-China-travel-related [11-13]. Of the four reported deaths (January 9, 2020-January 19, 2020), all were in China with the ages ranked between 61 to 89 years $[9,11,14,15]$. For the majority of the reported cases, the history of exposure to the Wuhan's Huanan Seafood Wholesale Market or other live markets is unknown [13]. Nevertheless, on January 19, 2020, first COVID-19-infected case of 35-year-old man presented to an urgent care clinic in Snohomish County, Washington was detected and reported in the United States [16].

\section{Case Definition for Surveillance (As of January 17, 2020)}

\section{Suspected Case Requiring Diagnostic Testing}

Patientswithacute respiratory infection who havesudden onset of at least one of the following symptoms : cough, sore throat, shortness of breath requiring hospitalization or not. In 14 days prior to onset of symptoms, met at least one of the following epidemiological criteria: 1) Were in close contact with a confirmed or probable case of COVID-19 infection; or 2) Had a history of travel to areas with presumed ongoing community transmission of COVID-19; or 3) Worked in or attended a health care facility where patients with COVID-19 infections were being treated [17].

\section{Close Contact}

Close contact is defined as : 1) Healthcare associated exposure, including providing direct care for patients with COVID-19 infection, working with healthcare workers infected with novel coronavirus, visiting patients or staying in the same close environment as a COVID-19 patient ; 2) Working together in close proximity or sharing the same classroom environment with a COVID-19 patient ; 3) Travelling together with a COVID-19 patient in any kind of conveyance ; and 4) Living in the same household as a COVID-19 patient. The epidemiological association may have occurred within a 14-day period before or after the onset of illness in the case under consideration [17].

\section{Probable Case}

The probable case is a suspected case for whom testing for COVID-19 is inconclusive by the result of the test reported by the laboratory section or for whom testing was positive on a pan-coronavirus assay [17].

\section{Confirmed Case}

The confirmed case is a individual with laboratory confirmation of COVID-19 infection, irrespective of clinical manifestations [17].

\section{Criteria to Initiate Testing for COVID-19}

Prompt case confirmation is essential for rapidly ensuring and effectively contact tracing, implementation of infection prevention and control measures according to national recommendations, and collection of relevant epidemiological and clinical data. Any individual fulfilling the criteria for a suspected case should be tested for COVID-19 infection. The laboratory test should be initiated immediately [17].

\section{Epidemic Trend by Mathematical Modelling}

Mathematical modeling for the epidemic trend of the COVID-19 outbreak in China conducted by Shen, et al. demonstrated that the national epidemic of COVID-19 may lead to at least a total of 8,042 (95\% Confidential Interval (CI) : 4,199-11,884) infections and at least 898 (95\% CI : $368-1,429)$ deaths, equivalent to a fatality rate of $11.02 \%$ (95\% CI : 9.26-12.78 \%) [18]. This fatality rate is lower than the rates of the Middle-East Respiratory Syndrome (MERS, $34.4 \%$ ) [19] and the Severe Acute Respiratory Syndrome (SARS, 14-15 \%) [20], indicating that COVID-19 may be a less virulent strain among the coronavirus family [18]

When the epidemic of COVID-19 in China started on December 12, 2019, the basic reproductive number (R0) of COVID-19 (an indication of the initial transmissibility of the virus) was estimated to be 4.71 (95\% CI: 4.50-4.92), whereas its effective reproductive number (Re) has decreased to 2.08 (95 \% CI: 1.99-2.18) as of January 22, 2020 [18]. With the assumption of no resurges of COVID-19 epidemic and 


\section{Open Access Journal of Pulmonary \& Respiratory Sciences}

the continually declining trend, Re will decrease below one within three months (77 (95\% CI: 75-80) days) of the epidemic initiation, indicating that the COVID-19 epidemic will gradually die off after this time [18]. In comparison with MERS and SARS, R0 of COVID-19 was similar to MERS in Jeddah (95 \% CI: 3.5-6.7) and Riyadh (95 \% CI : 2.0-2.8), Kingdom of Saudi Arabia, in 2014 [21] and SARS (R0 = 4.91) in Beijing, China, in 2003 [22]. Nevertheless, Zhao et al concluded that the mean estimate of R0 for the COVID-19 ranges from 2.24 (95\% CI : 1.96-2.55) to 3.58 (95\% CI : 2.89-4.39), and significantly larger than 1 if the reporting effort has been increased by a factor of between 8 - and 2-fold after the diagnostic protocol released on January 17, 2020 and several medical sup[plies reached Wuhan, and indicates the potential of COVID-19 to cause outbreaks [23].

\section{Laboratory Testing Methodology}

Rapid collection of the following specimens should be considered : when possible, specimens from both lower and upper respiratory tracts should be collected. For lower respiratory tract: expectorated sputum, endobronchial aspirate, and bronchoalveolar lavage. For upper respiratory tract: nasopharyngeal aspirate or nasal wash, oropharyngeal swab, and nasopharyngeal swab. The additional specimens for later testing are : when serological testing is available (serum : acute and convalescent specimens-possibly 2-4 weeks after acute phase). The other additional specimens for later testing are blood, urine, and feces [17].

Currently, the specific test recommended by the World Health Organization (WHO) for the diagnosis and confirmation of COVID-19 is real-time Reverse Transcriptase-Polymerase Chain Reaction (RT-PCR) supported by the study on detection of COVID-19 infection that conducted by Corman, et al. [24]. A single positive test should be confirmed by a second RTPCR assay targeting a different COVID-19 gene. A single negative COVID-19 test, particularly if specimens obtained from upper respiratory tract or a positive test result for another respiratory pathogen, indicates that the result does not exclude COVID-19 infection. If there is a strong suspicion for COVID-19 infection, another specimen should be tested with primary and secondary RT-PCR assays. When possible, sequence information should be generated from positive specimens [17].

\section{Discussion}

By January 2, 2020, Huang et al studied on clinical features of 41 admitted hospital patients with laboratory confirmation of COVID-19 infection revealed that the common symptoms at onset of illness were fever ( 40 of $41,98 \%$ ), cough (31 of $41,76 \%$ ), and fatigue or myalgia (18 of $41,44 \%$ ). The less common symptoms were sputum production $(11$ of 39,28

Attapon Cheepsattayakorn and Ruangrong Cheepsattayakorn. Acute Respiratory Syndrome and Pneumonia caused by COVID-19: A Systematic Review and Meta-Analysis. J Pulmon Respir Sci 2020, 5(1): 000126.
$\%$ ), headache ( 3 of $38,8 \%$ ), hemoptysis ( 2 of $39,5 \%$ ), and diarrhea ( 1 of $38,3 \%$ ). Dyspnea occurred in 22 of 40 patients (55\%) with median time from illness onset to dyspnea 8.0 days (Interquatile Range (IQR) : 5.0-13.0). Twenty-six of 41 patients (63\%) demonstrated lymphopenia. All 41 patients had pneumonia with pulmonary infiltrates on the chest computerized tomography. The complications included acute respiratory distress syndrome (12, $29 \%)$, RNAaemia $(6,15 \%)$, acute cardiac injury (5, $12 \%)$, and secondary infection $(4,10 \%)$. In comparison with non-ICU patients, ICU patients had higher plasma levels of interleukin (IL)-2, IL-7, IL-10, Granulocyte-Colony Stimulating Factor (GCSF), Interferon Gamma-Induced Protein10 (IP-10), Monocyte Chemoattractant Protein-1 (MCP-1), Monocyte Inflammatory Protein-1A (MIP-1A), and tumor necrosis factor (TNF)-alpha [25]. Among 41 hospitalized patients, most of the infected patients were men (30 of $41,73 \%$ ); less than $50 \%$ of the patients had underlying diseases (13 of $41,32 \%$ ), including diabetes mellitus ( 8 of $41,20 \%$ ), hypertension ( 6 of $41,15 \%$ ), and cardiovascular disease ( 6 of 41, $15 \%$ ). The median age was 49.0 years (IQR : 41.0-58.0). Twenty-seven of 41 patients had been exposed to Huanan seafood market [25]. Both SARS$\mathrm{CoV}$ and MERS-CoV were believed to originate in bats, these pathogens were transmitted directly to human from market civets and dromedary camels, respectively [26]. In 2013, a study [27] demonstrated the whole genome sequence of a SARs-like coronavirus in bats with ability to use human Angiotensin-Converting Enzyme 2 (ACE2) as a receptor for replication potentials in human cells [28]. Reliable rapid pathogen tests and feasible differential diagnosis based on clinical description are critical for clinicians in their first contact with suspected COVID-19-infected patients. A key uncertainty of this outbreak is when it started. Surveillance in China and elsewhere only began once the outbreak was identified in Wuhan, China. Had the outbreak began before January 2020 and in early January 2020, both domestically and internationally. If a substantial proportion of infection has been due to multiple exposures to various animals. These currently available data may represent a period of high transmission that will not be sustained over long periods of time. There is much uncertainty in both scale of the outbreak and key epidemiological information of transmission due to still being in the early days of this outbreak. Shen et al predicted the epidemiological trend COVID-19 by the mathematical modelling method and demonstrated a fatality rate of $11.02 \%$ [18], whereas the Chinese Center for Disease Control and Prevention reported that the confirmed case fatality rate is approximately $2.3 \%$, based on 1,023 deaths amongst 44,415 laboratory-confirmed cases, as of February 11, 2020 [29]. By observation in outbreaks of either MERS$\mathrm{CoV}$ or SARS, the rapidity of the growth of cases since the outbreak recognition is much greater, indicating that control or containment of this pathogenic organism may be much more difficult. In consideration of antiviral therapy, first

Copyright $(\subset)$ Attapon Cheepsattayakorn and Ruangrong Cheepsattayakorn. 


\section{Open Access Journal of Pulmonary \& Respiratory Sciences}

COVID-19-infected case reported in the United States [16], intravenous remdesivir (a novel nucleoside analogue prodrug in development $[30,31]$ ) was administrated on the evening of day 7 of hospitalization due to the patient's ongoing fevers, the persistent positive COVID-19 RNA at multiple sites, and notification of rales in both lungs at a period consistent with the development of roentgenographic pneumonia. On hospital day 8 (illness day 12), there is improvement of the patient's clinical condition. As of January 30, 2020, the patient is afebrile and has resolution of all symptoms, except his cough, that is decreasing in severity [16]. Another recent study on COVID-19 inhibition in vitro by remdesivir and chloroquine demonstrated that these drugs were highly effective in the control of COVID-19 infection in vitro [32]. These drugs should be evaluated in humans infected with COVID-19.

\section{Conclusion}

With bases on the genetic similarities between COVID-19 and SARS-CoV, the limited epidemiological data available from China and the case detection through entry screening outside of China, we hypothesize that new cases will be detected among travelers from Wuhan, China and from other countries outside China. Without implementation of proper infection prevention and control measures at the point of care for individuals under investigation, there will be a likelihood of disease outbreaks, particularly via traveler transmission, transmission on aircrafts, and healthcarerelated transmission in the destination countries. Further investigations for COVID-19 are urgently needed due to current global outbreaks and its potentially global health threat. Nevertheless, in the global experiences, systematic implementation of infection prevention and control measures were effective in controlling both SARS-CoV and MERS-CoV.

\section{Authors Contributions}

Dr. Attapon Cheepsattayakorn conducted the study framework and wrote the manuscript. Associate Professor Dr. Ruangrong Cheepsattayakorn contributed to scientific content and assistance in manuscript writing. Both authors read and approved the final version of the manuscript.

\section{Competing Interests}

The authors declare that they have no actual or potential competing financial interests.

\section{Funding Sources}

The authors disclose no funding sources.

\section{References}

1. Yin Y, Wunderlink RG (2018) MERS, SARS and other coronaviruses as causes of pneumonia. Respirology 23(2): 130-137.

2. OAG (2020) Traffic Analyser FAQs. Luton.

3. Bogoch II, Watts A, Thomas-Bachli A, Huber C, Kraemer MU, et al. (2020) Pneumonia of Unknown Etiology in Wuhan, China: Potential for International Spread via Commercial Air Travel. Journal of Travel Medicine, pp: 1-3.

4. WHO (2020) WHO Statement Regarding Cluster of Pneumonia Cases in Wuhan, China.

5. New coronavirus pneumonia latency (2020).

6. Wuhan City Health Committee (2019) Wuhan Municipal Health and Health Commission's briefing on the current pneumonia epidemic situation in our city.

7. National Health Commission of the People's Republic of China (2020) Epidemic situation of new coronavirus infection on January 21.

8. WHO Regional Office for Western Pacific (WOWPRO) Novel Coronavirus (2019-nCoV).

9. Wuhan City Health Committee (WCHC) (2020) Wuhan Municipal Commission on Health on pneumonia of new coronavirus infection (January 21, 2020).

10. Health Commission of Guangdong Province (2020) Our province actively responds to pneumonia epidemic of new coronavirus infection.

11. Wuhan City Health Committee (WCHC) (2020) Wuhan Municipal Commission of Health and Health on pneumonia of new coronavirus infection.

12. European Center for Disease Prevention and Control (ECDC) (2020) Rapid Risk Assessment : Cluster of pneumonia cases by a novel coronavirus, Wuhan, China, 2020

13. Xinhua Net (2020) Xi orders resolute efforts to curb virus spread.

14. Wuhan City Health Committee (WCHC) (2020) Experts explain the latest bulletin of unknown cause of viral pneumonia.

15. Wuhan City Health Committee (WCHC) (2020) Wuhan 


\section{Open Access Journal of Pulmonary \& Respiratory Sciences}

Municipal Commission of Health on pneumonia of new coronavirus infection (January 20, 2020).

16. Holshue ML, DeBolt C, Lindquist S, Lofy KH, Wiesman J, et al. (2020) First case of 2019 novel coronavirus in the United States. N Engl J Med 382: 929-936.

17. European Centre for Disease Prevention and Control (2020) Case definition and European surveillance for COCID-19.

18. Shen M, Peng Z, Xiao Y, Zhang L (2020) Modelling the epidemic trend of the 2019 novel coronavirus outbreak in China. BioRxiv (The preprint server for biology).

19. World Health Organization (2019) MERS monthly summary, November 2019.

20. World Health Organization (2003) Update 49-SARS case fatality ratio, incubation period.

21. Majumder MS, Rivers C, Lofgren E, Fishman D (2014) Estimation of MERS-Coronavirus reproductive number and case fatality rate for the Spring 2014 Saudi Arabia outbreak: Insights from publicly available data. PLoS Curr pp: 1-6.

22. Gumel AB, Ruan SG, Day T, Watmough J, Brauer F, et al. (2004) Modelling strategies for controlling SARS outbreaks. Proc Biol Sci.271(1554): 2223-2232.

23. Zhao S, Lin Q, Ran J, Musa SS, Yang G, et al. (2020) Preliminary estimation of the basic reproduction number of novel coronavirus (2019-nCoV) in China, from 2019 to 2020: a data-driven analysis in the early phase of the outbreak. Int J Infect Dis 92: 214-217.

24. Corman VM, Landt O, Kaiser M, Molenkamp R, Meijer A, et al. (2020) Detection of 2019 novel coronavirus (2019nCoV) by real-time RT-PCR. Euro Surveill 25(3).

25. Huang C, Wang Y, Li X, Ren L, Zhao J, et al. (2020) Clinical features of patients infected with 2019 novel coronavirus in Wuhan, China. Lancet 395(10223): 497-506.

26. Cui J, Li F, Shi ZL (2019) Origin and evolution of pathogenic coronaviruses. Nat Rev Microbiol 17(3): 181192.

27. Ge XY, Li JL, Yang XL, Chmura AA, Zhu G, et al. (2013) Isolation and characterization of a bat SARS-like coronavirus that uses the ACE2 receptor. Nature 503(7477): 535-538.

28. Wang M, Hu Z (2013) Bats as animal reservoirs for the SARS coronavirus: hypothesis proved after 10 years of virus hunting. Virol Sin 28(6): 315-317.

29. The novel coronavirus pneumonia emergency response epidemiology team, the epidemiological characteristics of an outbreak of 2019 novel coronavirus disease (COVID-19), China 2020.

30. Mulangu S, Dodd LE, Davey RT, Mbaya OT, Proschan M, et al. (2019) A randomized, controlled trial of ebola virus disease therapeutics. N Engl J Med 318(24): 2293-2303.

31. Sheahan TP, Sims AC, Leist SR, Schafer A, Won J, et al. (2020) Comparative therapeutic efficacy of remdesivir and combination lopinavir, ritonavir, and interferon beta against MERS-CoV. Nat Commun 11(1): 222.

32. Wang M, Cao R, Zhang L, Yang X, Liu J, et al. (2020) Remdesivir and chloroquine effectively inhibit the recently emerged novel coronavirus (2019-nCoV) in vitro. Cell Research 30: 269-271. 